\title{
A cryogen-free HTS Josephson junction detector for terahertz imaging
}

J. Du ${ }^{(1)}$, K. Smart ${ }^{(1)}$, L. Li ${ }^{(1)}$, K. E. Leslie ${ }^{(1)}$, S. M. Hanham ${ }^{(2)}$, D. H. C. Wang ${ }^{(1)}$, C. P. Foley ${ }^{(1)}$, F. Ji ${ }^{(3)}$, X. D. Li ${ }^{(3)}$, and D. Z. Zeng ${ }^{(3)}$

(1) Commonwealth Scientific and Industrial Research Organisation (CSIRO), Lindfield, NSW 2070, Australia

(2) Dept. of Materials, Imperial College, London, U.K.

(3) Chengdu Shuguang Optical Fiber Network Co., Ltd, Chengdu, Sichuan, China

\begin{abstract}
A cryogen-free terahertz ( $\mathrm{THz}$ ) imaging system based on a high-temperature superconducting (HTS) Josephson junction detector is reported. The detector was made of $\mathrm{YBa}_{2} \mathrm{Cu}_{3} \mathrm{O}_{7-\mathrm{x}}(\mathrm{YBCO})$ step-edge Josephson junction and integrated into an on-chip thin-film antenna. The HTS Josephson detector was cooled via a commercial mechanical cryocooler; an important step towards cryogen-free $\mathrm{THz}$ instrumentation, which is critical for industrial acceptance. In addition, it is shown that operating the detector in a cryocooler provides improved flexibility for optimising the detector parameters and performance due to the ability to adjust the temperature compared to liquid nitrogen cooling methods. The DC and AC characteristics, the detector responsivity and the noise-equivalent power (NEP) of the detector, and resulting image quality were studied as the function of operating temperatures.
\end{abstract}

\section{Introduction}

Terahertz ( $\mathrm{THz}$ ) radiation lies in the frequency gap between the microwave and infrared (IR) regions of the electromagnetic spectrum and displays many unique properties such as strong sensitivity to water, high transmission through a range of non-conducting materials and spectroscopic responses to materials $[1,2]$. These features mean THz technology holds significant potential in the areas of security screening (detection of explosives, drugs, hidden weapons and other objects), material characterisation, non-destructive testing, medical imaging, chemical sensing and food and agricultural product inspection (see, for example, review papers [1-4]). THz radiation is non-ionising and is therefore safer for humans compared to X-ray based imaging. Despite huge potential, the uptake of $\mathrm{THz}$ technology has been slow mainly due to limitations of available source and detector 
technologies. In recent years, $\mathrm{THz}$ science and technology has become an intensely researched field around the world and major progress has been made in developing new $\mathrm{THz}$ sources, detectors, imaging and spectroscopy systems using a range of technologies.

Superconducting devices offer a viable option as they are excellent generators and detectors of terahertz radiation. Superconducting THz emitters [5, 6] and sensors [7-12] have been reported. We have, in recent years, developed high- $T_{c}$ superconducting (HTS) Josephson $\mathrm{THz}$ detectors and demonstrated their use for THz imaging [10-12]. HTS devices offer not only the obvious advantage of higher operating temperatures compared to their low- $T_{c}$ superconducting (LTS) counterpart, but also a very high band-gap voltage which extends their response well into the $\mathrm{THz}$ range. In previous work, the HTS detectors were operated in liquid helium [9] or liquid nitrogen [10, 11]. In this work, we have developed a terahertz imaging system based on a HTS Josephson detector operating in a commercial cryocooler. A cryogen-free system is an essential step towards industrial acceptance. Compared to LTS detectors, HTS detectors can operate using a relatively inexpensive and more compact single-stage cryocooler. Furthermore, the controllable temperature of a cryocooler allows for the optimisation of the detector parameters to achieve improved performance. This work also differs from our previous system in that we have integrated a more compact solid-state $\mathrm{THz}$ source and redesigned the thin-film antenna-coupled HTS detectors. We shall show that THz images of highvisual quality were obtained with this configuration. The full characterisations of the detectors operating in the cryocooler and the acquired $\mathrm{THz}$ image quality at different temperatures are described in this paper.

\section{Experimental Details}

\section{$\underline{\text { About the detectors }}$}

The terahertz detectors consist of a HTS step-edge Josephson junction integrated across the slot of a ring-slot antenna which was designed with a centre frequency of $600 \mathrm{GHz}$ and $~ 12 \%$ bandwidth, shown in Fig. 1c. In operation, an incident THz wave (RF signal) excites currents on the antenna which pass through the junction; the RF signal is detected via changes in the junction's electrical characteristics. A more detailed description of the operating principle of this type of detector can be found in reference [11].

The HTS detectors were fabricated on MgO substrates using the CSIRO HTS step-edge junction technology $[13,14]$. Briefly, the MgO substrates were photo-lithographically patterned and etched using Ar-ion beam milling to produce steps of $\sim 400 \mathrm{~nm}$ in height with an angle of $\sim 35^{\circ}$ to the substrate surface. S-type (smooth film morphology) YBCO films of $220 \mathrm{~nm}$ thickness and a $50 \mathrm{~nm}$ in 
situ gold film were deposited by Ceraco GmbH, Germany. The YBCO film is patterned and ion-beam etched to form a $2 \mu \mathrm{m}$ wide Josephson junction. The in situ Au was removed from the junction area using the in-housing Ar reactive ion etching technique. An additional gold layer of $\sim 300 \mathrm{~nm}$ thick was then deposited and patterned to form the antenna structure and contact pads. An in situ Au layer is necessary to improve the adhesion of the Au layer to YBCO film and reduce the contact resistance [15]. However, our study also showed if using YBCO film and Au bi-layer for the antenna, the noise increased presumably due to both increased surface resistance of YBCO film at high frequency and large contact resistance between the $\mathrm{YBCO}$ and $\mathrm{Au}$ films. We have, therefore, used $\mathrm{Au}$ thin-film for the antenna and kept only narrow YBCO micro strip lines for the DC current and voltage leads (see Fig. 1 (c) and (d)).

Figure 1 shows a micrograph of the fabricated chip containing a $2 \times 2$ array of gold thin-film antennacoupled to YBCO Josephson junction detectors. The antennas are positioned on the centre of the substrate so that they are at the focal spot of a $6 \mathrm{~mm}$ diameter high resistivity silicon lens which is bonded to the reverse side of the substrate. The combination of the $\mathrm{MgO}$ substrate and hemispherical silicon lens forms a hyper-hemispherical lens which ensures a high antenna directivity and efficient coupling between free-space and the antennas. The arcs visible in Fig. 1a denote the extent of the lens and were used to align the lens to the antennas.

The design of the antenna-coupled detector is different from those reported in $[10,11]$ where the junction was located across the ring slot but at a position orthogonal to the axis of the coplanar waveguide filter. The step-edge junctions reported here are integrated across the ring slot at a position which is co-linear with the coplanar waveguide filter and the local slot width has two different sizes: $4 \mu \mathrm{m}$ and $7 \mu \mathrm{m}$ for optimising the RF coupling. The slot width is widened at the junction for easier junction alignment in the ring slot during device fabrication. These modifications were undertaken to improve the impedance matching between the ring slot antenna and the junction. The very low impedance (typically, 1-5 $\Omega$ ) of the junction makes impedance matching difficult and care must be taken when designing the device to avoid shorting the ring slot antenna with the junction which would have a deleterious effect on the antenna's performance. 

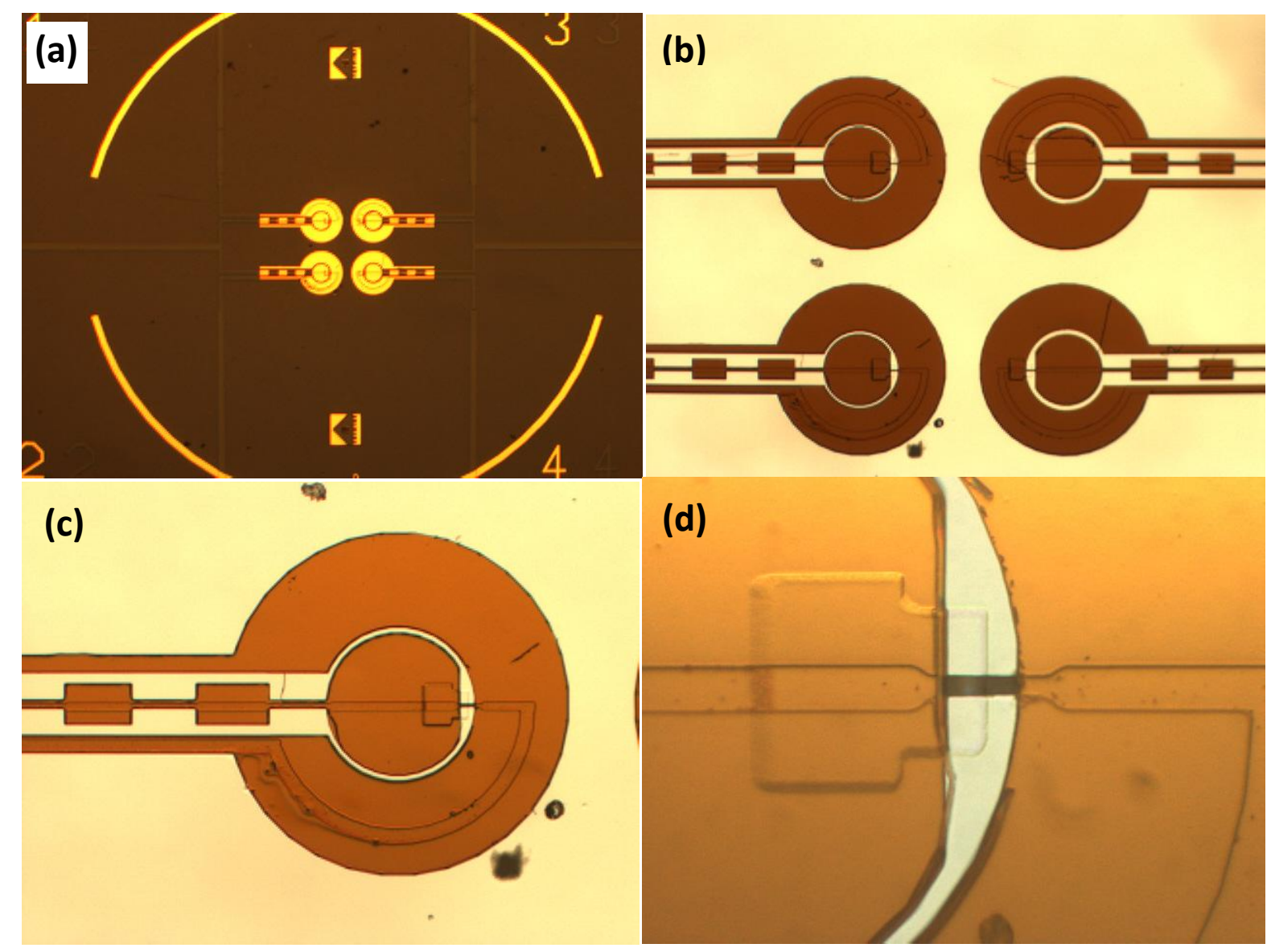

Figure 1: Micrographs of the fabricated device chip containing four antenna-coupled HTS Josephson detectors at magnifications (a) 100x, (b) 200x, (c) 500x and (d) 1000x. (d) shows a $2 \mu \mathrm{m}$ wide YBCO electrode (black colour) is patterned across the antenna slot connecting the inner and outer conductors of the antenna and across a step-edge on the MgO substrate inside the slot forming a step-edge junction.

The detector/antenna/lens was then mounted on a commercial pulse-tube cryocooler (PTC) (Janis model PTSHI-4-5 by Research Inc.) cold-head with the silicon lens on the back of the chip facing the cryocooler window where the $\mathrm{THz}$ beam radiation is incident, as shown in Fig. 2. The detector characteristics and acquisition of the THz images were studied over the operating temperature range $50 \mathrm{~K}$ to $77 \mathrm{~K}$; a temperature range attainable with a single stage miniature cryocooler. It should be noted that a significantly smaller cryocooler than the one used here could be employed in future systems to improve system portability.

\section{The THz imaging system}

Figure 2 shows a photograph of the THz imaging system integrated with the PTC cooler. It is an active transmission imaging scheme, i.e., the object being imaged is illuminated by a continuouswave $\mathrm{THz}$ source and the $\mathrm{THz}$ wave transmitted through the object is detected. A more compact commercial solid-state THz source (VDI 625 GHz Amplifier Multiplier Chain (AMC)) with a small 
economy frequency synthesizer as the RF input signal was used to replace the bulky back-waveoscillator (BWO) THz source used in previous experiments [10-12] for a more compact simpler electronic imaging system. The THz signal was transmitted via a quasi-optical scheme with two pairs of collimating and focusing mirrors onto the sample and then to the detector in the cryocooler. The cold stage was connected to the compressor via semi-rigid flexible gas cables clad with vibration damping materials. No $\mu$-metal or other low-frequency magnetic shields were used around the sample stage. The HTS Josephson detector operated stably without observable performance degradation (see later section for noise characterisation). The sample being imaged is raster scanned in a plane perpendicular to the beam axis using a linear XY motor stage. The theoretical spatial resolution can be estimated using the Rayleigh criterion $\Delta x=1.22 \lambda f / D$ assuming the perfect optical alignment, where the wavelength $\lambda=3 \times 10^{8} \mathrm{~ms}^{-1} / 614 \mathrm{GHz}=488 \mu \mathrm{m}, f$ and $D$ are focal length and diameter of the focusing mirror. The estimated theoretical spatial resolution is $1.19 \mathrm{~mm}$. In the experiments, we scanned the sample at a typical resolution of $0.5 \mathrm{~mm}$. An area of $5 \mathrm{~cm} \times 5 \mathrm{~cm}$, i.e. an image size of $100 \times 100$ or 10,000 pixels took about 10 minutes to complete.

The Josephson junction was current biased above its critical current $I_{\mathrm{c}}$ at the point on the DC currentvoltage characteristic (IVCs), where the dynamic resistance, $R_{\mathrm{d}}=\mathrm{dV} / \mathrm{d} l$, is the maximum. The voltage response, $V_{\mathrm{RF}}$, induced by the chopper-modulated $\mathrm{THz}$ power was amplified 100 times using a low noise integrated circuit (Burr Brown type INA 103) at room temperature. A lock-in amplifier synchronized to the $1 \mathrm{kHz}$ optical chopper wheel signal was used to acquire the voltage response, which was processed using an in-house developed Labview software program to produce an image. The software PicoScope was used to capture the DC IVCs and the real-time voltage response traces. A HP 35670A dynamic signal analyser was used to record the detector noise spectra.

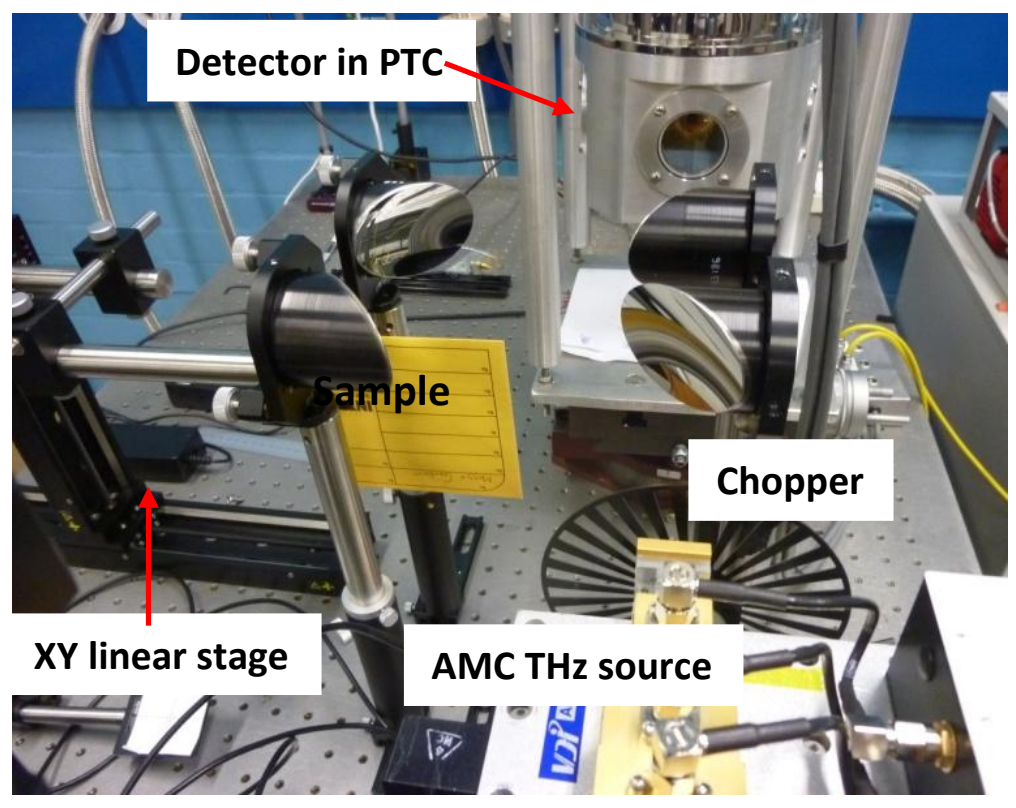


Figure 2: Photograph of the transmission THz imaging system: a solid-state $\mathrm{THz}$ source on the front, an optical chopper, a sample driven by a motorised XY-linear stage, and the antenna-coupled HTS detector mounted on the cold-head facing the window on a commercial PTC cooler on the back.

\section{Results and discussion}

\section{Detector Characterisations}

Figure 3 shows the IVCs of a detector at different operating temperatures. The junction demonstrated a resistively-shunted-junction like behaviour with a normal resistance of $R_{\mathrm{n}} \approx 1.5 \Omega$. The junction critical current, $I_{c}$, increased with decreasing temperature, from $\sim 80 \mu \mathrm{A}$ at $77 \mathrm{~K}$ to 827 $\mu \mathrm{A}$ at $50 \mathrm{~K}$. The junction characteristic voltage, $V_{\mathrm{c}} \equiv I_{\mathrm{c}} R_{\mathrm{n}}$, versus the temperature is plotted in the inset. The $I_{\mathrm{c}} R_{\mathrm{n}}$ value increases from $\sim 120 \mu \mathrm{V}$ at $77 \mathrm{~K}$ to $\sim 1.24 \mathrm{mV}$ at $50 \mathrm{~K}$, which corresponds to a characteristic frequency, $f_{\mathrm{c}} \equiv I_{\mathrm{c}} R_{\mathrm{n}} / \Phi_{0}$ (where $\Phi_{0}$ is the magnetic flux quantum, $1 / \Phi_{0} \equiv 2 \mathrm{e} / \mathrm{h}=$ $0.4836 \mathrm{GHz} / \mu \mathrm{V}$ ) of $\sim 60 \mathrm{GHz}$ at $77 \mathrm{~K}$ to $\sim 600 \mathrm{GHz}$ at $50 \mathrm{~K}$. By varying the temperature, the $V_{\mathrm{c}}$ and $f_{\mathrm{c}}$ values can be adjusted to optimise the detector performance or achieve broadband frequency detection, demonstrating the advantage of using a temperature-adjustable cryocooler.

Figure 4 (a) shows the IVCs at $70 \mathrm{~K}$ under THz signal radiation at different power levels. The variation of the power level was achieved by inserting layers of cardboard (CB) of which each layer was measured to exhibit $3 \mathrm{~dB}$ of attenuation. The $\mathrm{THz}$ signal suppresses the $I_{\mathrm{c}}$ value and induces Shapiro steps at voltages $V_{n}=n \Phi_{0} f_{s}=n \times(1.27 m V)(n=\ldots,-2,-1,0,1,2, \ldots)$, where the signal frequency $f_{s}=$ 614.2 $\mathrm{GHz}$. The relationship of the junction critical $I_{\mathrm{c}}$ value and the 1st Shapiro step height versus the $\mathrm{THz}$ signal power are plotted out in Fig. $4(\mathrm{~b})$. The $I_{\mathrm{c}}$ decreases and first Shapiro current step increases with increasing the $\mathrm{THz}$ signal power as predicted. By measuring the current step heights, we could estimate the $\mathrm{THz}$ power coupled into the junction using the Bessel functions (in approximation): $I_{d} I_{c 0}=J_{0}\left(2 e V_{s} / h f\right)$ or $\Delta I_{n=1}=J_{1}\left(2 e V_{s} / h f\right)$, where the $J_{0}$ and $J_{1}$ are 0 and 1-order Bessel functions, $I_{c 0}$ is the junction critical current without THz radiation, and $P_{\mathrm{s}}=1 / 2 \mathrm{~V}_{\mathrm{s}}^{2} / R_{\mathrm{n}}$ or $=1 / 2 I_{\mathrm{s}}^{2} R_{\mathrm{n}}$ is the RF signal power coupled into the junction. The $\mathrm{THz}$ power coupled into the junction was estimated to be in the range of sub micro watts to several micro watts. 


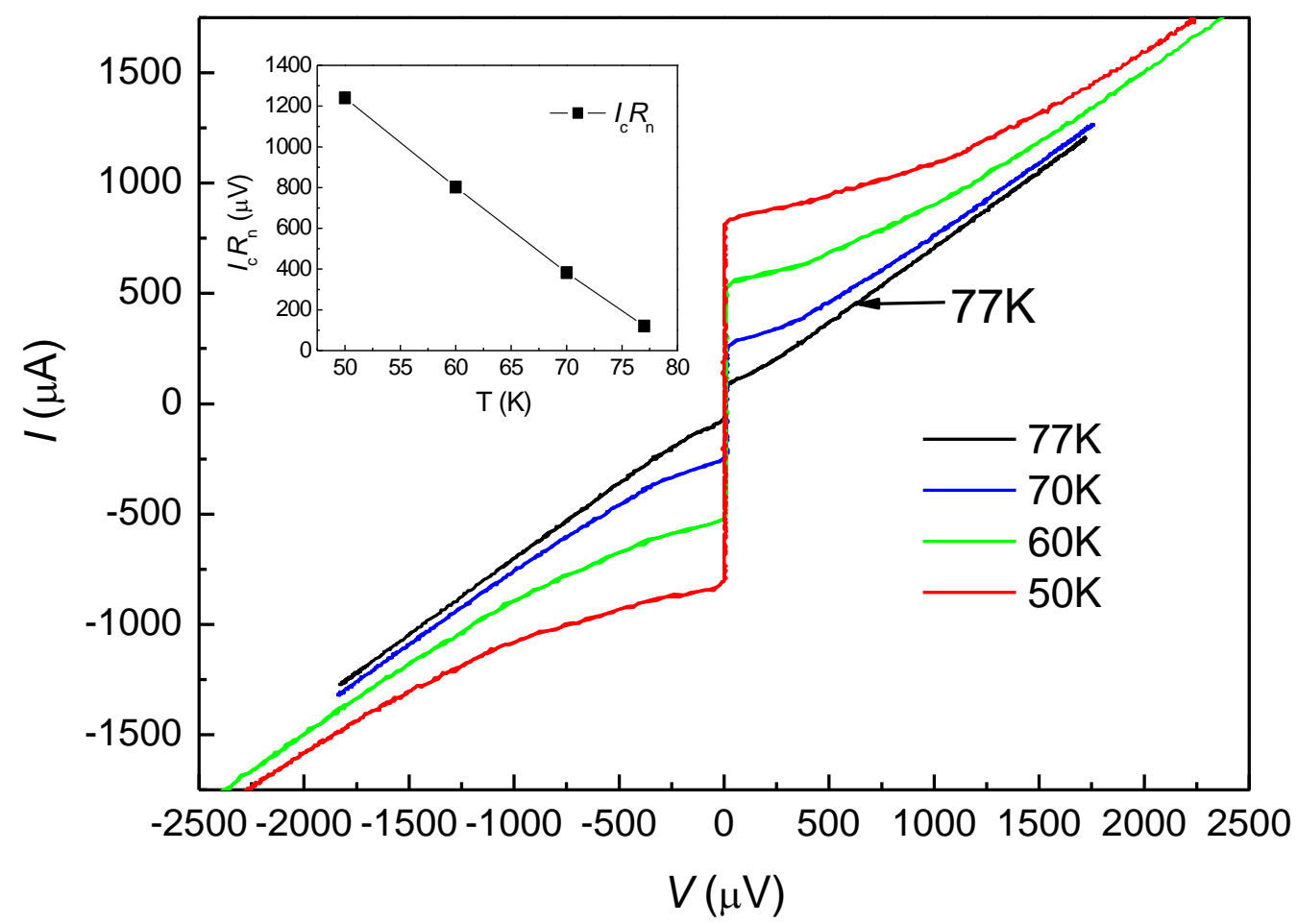

Figure 3: DC IVCs at different temperatures; inset shows the relationship of $I_{\mathrm{c}} R_{\mathrm{n}}$ product versus $T(\mathrm{k})$. 

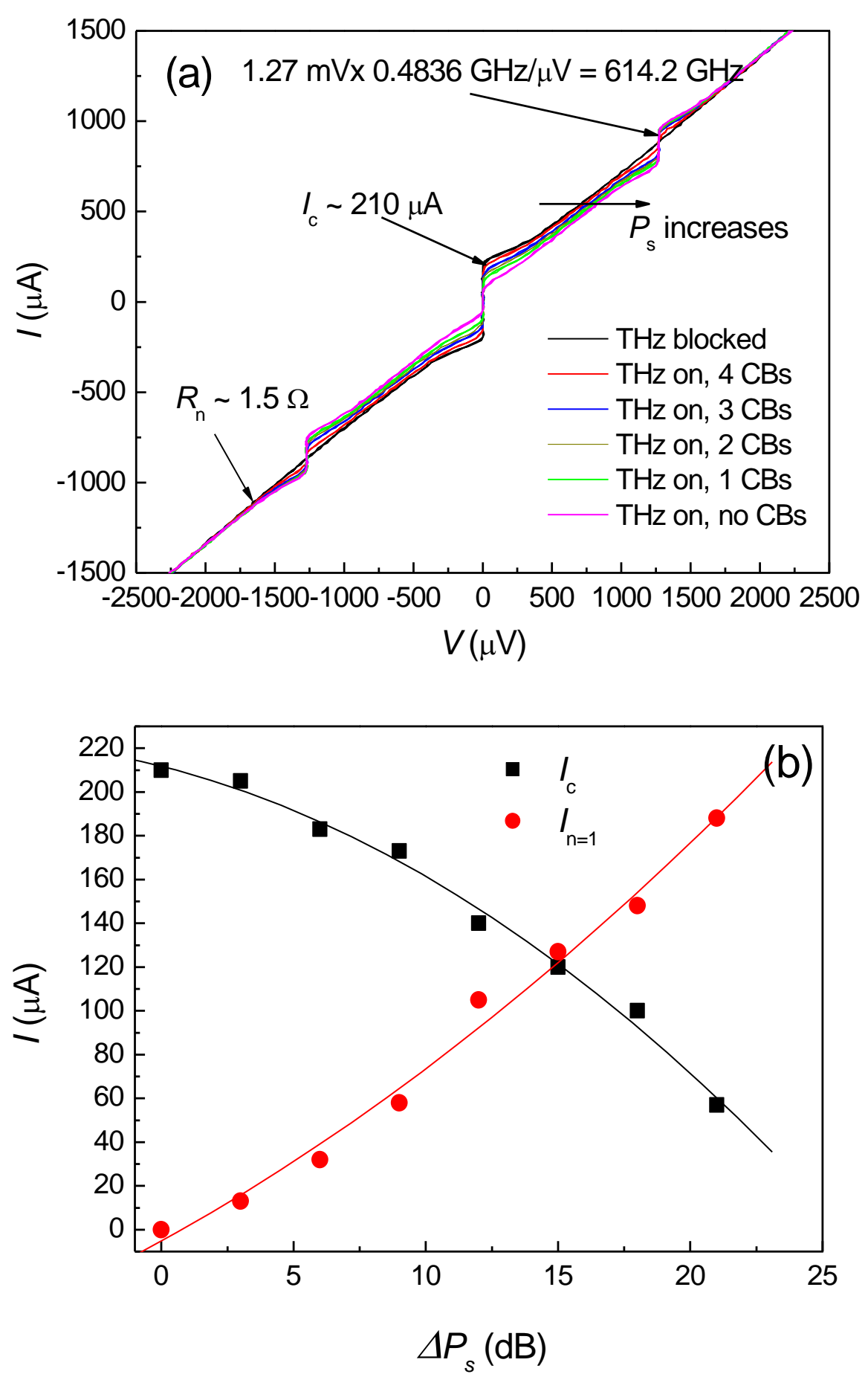

Figure 4 (a) The IVCs at $70 \mathrm{~K}$ versus applied THz signal power; (b) shows the relationship of the junction critical $I_{c}$ value and the current height of the $1^{\text {st }}$ Shapiro step versus with the $\mathrm{THz}$ signal power changes (the lines through the symbols are guide to the eye). 


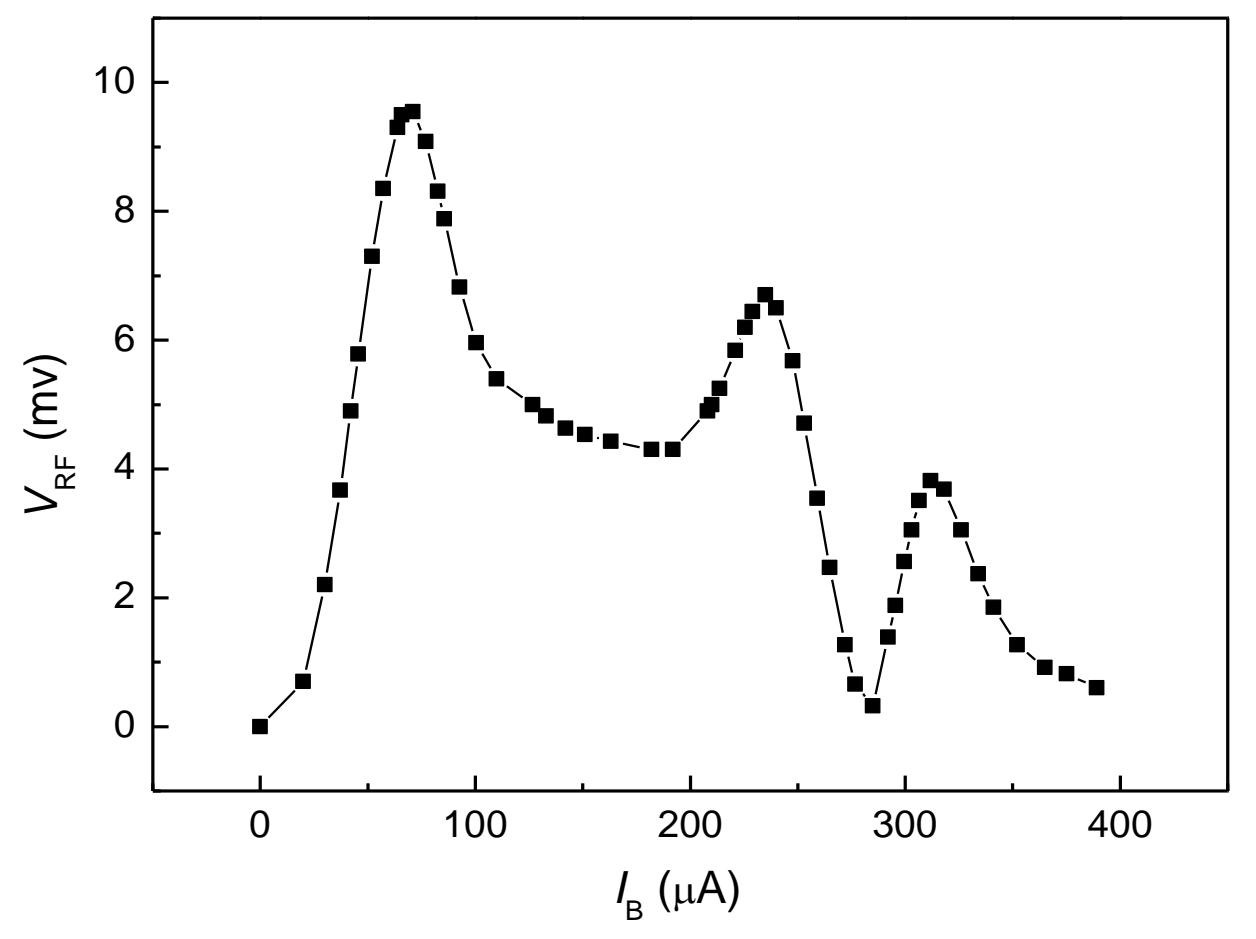

Figure 5: The measured voltage response $V_{\mathrm{RF}}$ versus the bias current $I_{\mathrm{B}}$ at $70 \mathrm{~K}$. The maximum output voltage occurs at the point just above the THz signal suppressed $I_{\mathrm{c}}$.

When the Josephson junction is current biased above its critical current, $I_{c 0}$, on the I-V curve and a chopper-modulated THz wave is incident, a RF voltage response, $V_{R F}$, is induced across the junction. The voltage response $V_{R F}$ amplitude is bias-current dependent. Fig. 5 shows the measured voltage response $V_{R F}$ versus the bias current $I_{B}$ at $70 \mathrm{~K}$. The voltage output changes with the bias position and reaches a maximum at the point just above the $\mathrm{THz}$ signal-suppressed $I_{\mathrm{c}}$.

We have measured the antenna-coupled detector RF voltage response versus the signal frequency. The obtained frequency response range is from $590 \mathrm{GHz}$ to $650 \mathrm{GHz}$, i.e. $10 \%$ bandwidth. However, we also measured the radiation frequency pattern of the AMC source using the VDI diode, which showed the similar frequency pattern. Therefore, we conclude that the simulated bandwidth ( 12\%) of the ring slot is larger than the source frequency bandwidth ( 10\%) and it was not possible for us to experimentally verify the radiation pattern bandwidth of the ring-slot antenna.

Figure 6 shows the acquired real-time voltage response $V_{R F}(t)$ to the chopped $\mathrm{THz}$ beam at different operating temperatures. The amplitude of the detector response increases significantly with decreasing the temperature. This is because the dynamic resistance $R_{\mathrm{D}}(\mathrm{V})=\mathrm{d} V / \mathrm{d} /$ increases with 
decreasing temperature and therefore the voltage response $V_{\mathrm{RF}}=R_{\mathrm{D}} \Delta l$ increases, where $\Delta l$ is the THz induced current change amplitude. The maximum amplitude of voltage response at $50 \mathrm{~K}$ is $122 \mathrm{mV}$ and the noise floor on the lock-in amplifier was $\sim 1 \mu \mathrm{V}$ (after $\times 100$ amplification), giving a signal-tonoise ratio (SNR) in an order of $\sim 1 \times 10^{5}$ or $100 \mathrm{~dB}$, which is further improvement from previously achieved SNR of $45 \mathrm{~dB}$ [11]. The voltage response amplitude decreases with increasing temperature reducing to $V_{\mathrm{RF}} \approx 5.7 \mathrm{mV}$ and $\mathrm{SNR} \sim 5 \times 10^{3}$ at $77 \mathrm{~K}$.

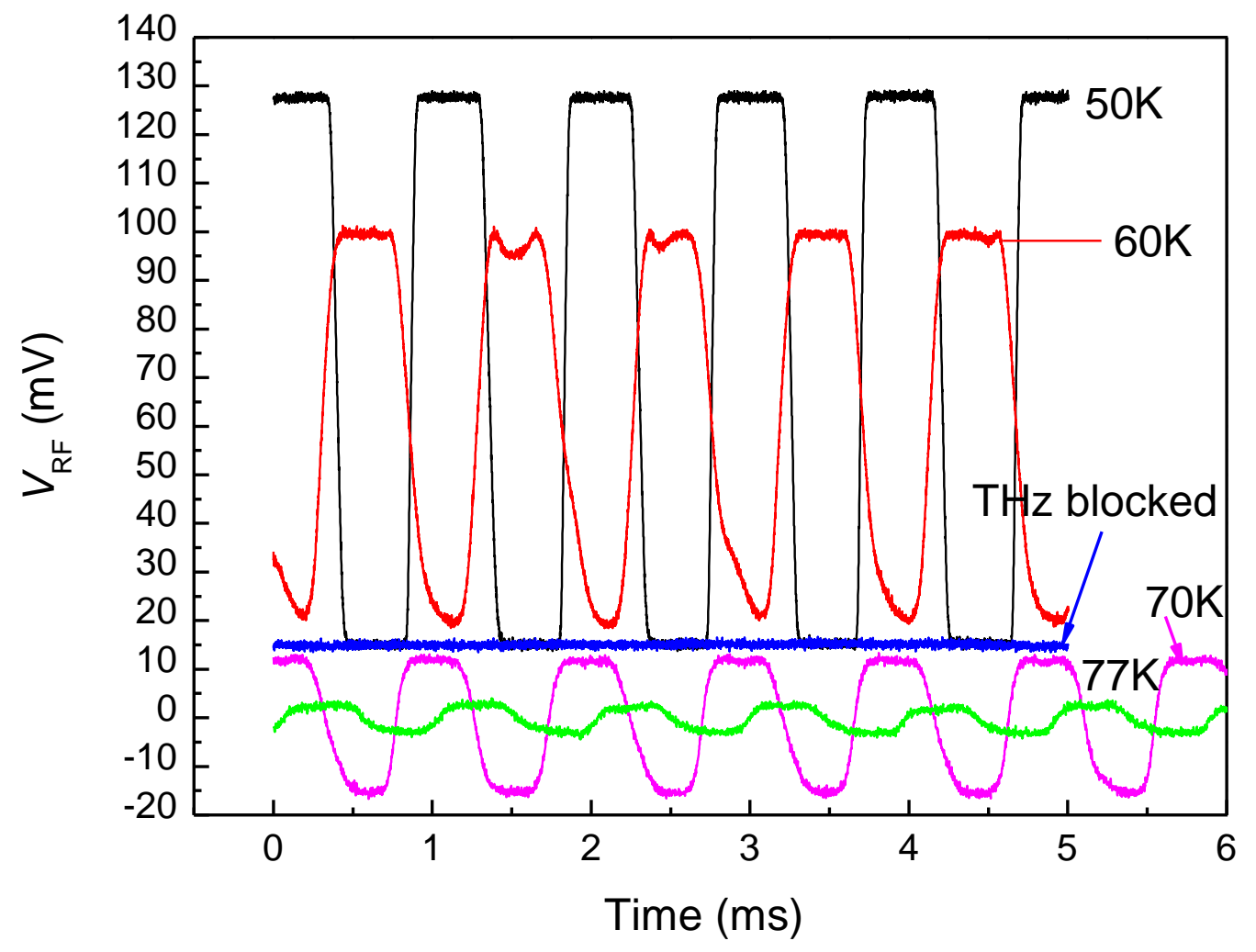

Figure 6: Measured real-time detector voltage signal at the 100x LNA output at different temperatures (DC offset has been added to some of the traces to improve clarity).

The voltage output for a given input RF signal will depend on the responsivity of the maximum zerovoltage current to RF signal. The greater the dynamic resistance, $R_{\mathrm{D}}=d V / d l$, at the operating point, the greater the output voltage will be. We can estimate the detector voltage responsivity using the following simple expression if we assume the load circuit is matched to the dynamic resistance, $R_{\mathrm{D}}$, of the junction and the input impedance to be simply the junction resistance $R_{\mathrm{n}}$ [16].

The voltage responsivity can be expressed as:

$$
\eta=V_{\mathrm{RF}} / P_{\mathrm{s}}=2 V_{\mathrm{RF}} / I_{\mathrm{s}}^{2} R_{\mathrm{n}}=R_{\mathrm{D}} /\left(2 I_{\mathrm{c} 0} R_{\mathrm{n}} \Omega^{2}\right)
$$


where $P_{\mathrm{s}}=(1 / 2) V_{\mathrm{s}}^{2} / R_{\mathrm{n}}$ or $(1 / 2) I_{\mathrm{s}}^{2} R_{\mathrm{n}}$ is the RF signal power coupled into the junction $\left(I_{\mathrm{s}}\right.$ and $V_{\mathrm{s}}$ are the $\mathrm{RF}$ signal current and voltage amplitude respectively), which induces the voltage output $V_{\mathrm{RF}} \Omega$ is the normalised frequency, $\Omega=f_{\mathrm{s}} / f_{\mathrm{c}}=f_{\mathrm{s}} /\left(I_{c 0} R_{n} \times 2 \mathrm{e} / \mathrm{h}\right)$ and $1 / \Phi_{0}=2 \mathrm{e} / \mathrm{h}=0.4836 \mathrm{GHz} / \mu \mathrm{V}$. Here $\Omega \geq 1$ when the detector operates in the range of $77 \mathrm{~K}$ to $50 \mathrm{~K}$. It can be seen from equation (1) that the responsivity is inversely proportional to $\Omega^{2}$ (or $f_{\mathrm{s}}^{2}$ ), i.e., lower frequency and/or higher $I_{\mathrm{c}} R_{\mathrm{n}}$ value raise the sensitivity. The $R_{\mathrm{D}}$ value can be obtained by differentiating the measured detector IVCS and the maximum $R_{\mathrm{D}}$ value was used to estimate the responsivity. Figure 7 shows the responsivity against the operating temperature and the $I_{\mathrm{c}} R_{\mathrm{n}}$ value. The responsivity increases significantly with decreasing temperature (thus increasing $I_{\mathrm{c}} R_{\mathrm{n}}$ value) from $77 \mathrm{~K}$ to $50 \mathrm{~K}$, where the responsivity $\eta \approx$ $7000 \mathrm{~V} / \mathrm{W}$ at $50 \mathrm{~K}$. This shows that operating the HTS Josephson detector in a cryocooler has the advantage of improving the detector performance significantly by adjusting the temperature compared to using liquid nitrogen ( $77 \mathrm{~K}) .50 \mathrm{~K}$ can be readily achieved using a single-stage miniature cryocooler. The achieved responsivity at $50 \mathrm{~K}$ is about an order of magnitude better than a commercial semiconductor diode detector in the same frequency band (for example, $750 \mathrm{~V} / \mathrm{W}$ for a VDI zero biased detector at 500-750 GHz [17]).

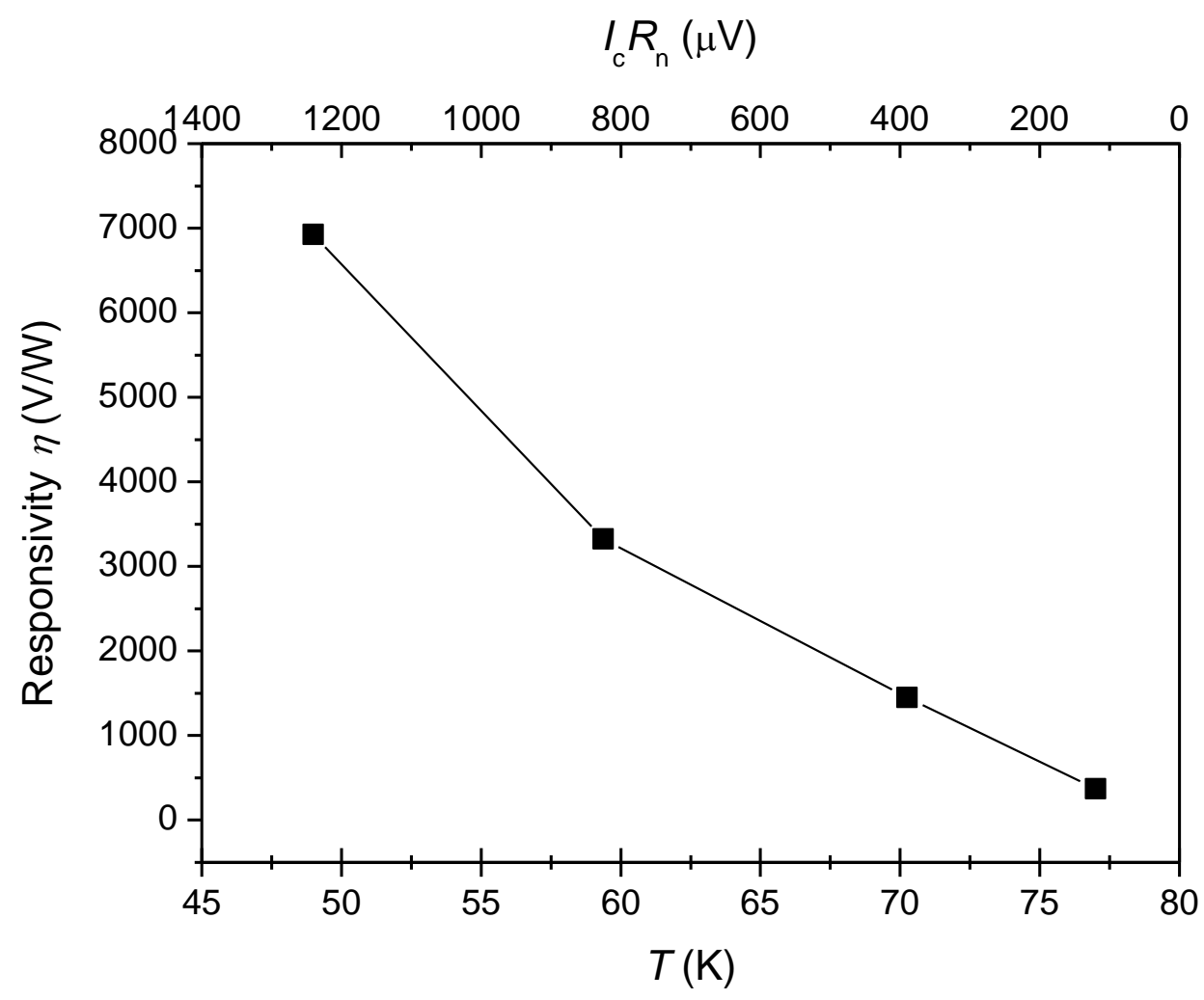

Figure 7: The detector voltage responsivity versus operating temperature and $I_{\mathrm{c}} R_{\mathrm{n}}$ value. 
We also measured the noise performance of the Josephson detectors. Figure 8 shows the noise spectra of the low-noise amplifier (LNA), detector base-noise with cryocooler compressor "off" and "on" when the THz signal is blocked. The measured LNA input noise when shorted at input is 2 $\mathrm{nV} / \sqrt{\mathrm{Hz}}$. When the detector is connected to the LNA, the input noise is $3-4 \mathrm{nV} / \sqrt{\mathrm{Hz}}$ of which the slight increase is believed to be due to the long wires from the detector to the LNA. There were negligible changes the noise floor when the PTC compressor turned "on" and "off" indicating that the cryocooler contributes little noise to the detector noise. The detector noise was limited by the room temperature LNA rather than the HTS Josephson junction intrinsic noise. We can estimate the noise equivalent power (NEP) of the detector using the input noise of the LNA and the measured voltage responsivity of the detector. A NEP of $\sim 3 \times 10^{-13} \mathrm{~W} / \sqrt{ } \mathrm{Hz}$ to $5 \times 10^{-12} \mathrm{~W} / \sqrt{\mathrm{Hz}}$ was obtained for the detector operating at $50 \mathrm{~K}$ to $77 \mathrm{~K}$, an order of magnitude improvement when the operating temperature lowered from $77 \mathrm{~K}$ to $50 \mathrm{~K}$. It is anticipated that the sensitivity and the NEP should continue to improve with further decreasing the temperature. However, our HTS detectors were designed for operating at higher temperatures and $50-77 \mathrm{~K}$ is the typical temperature range a singlestage cryocooler could reach. It is worth mentioning that the estimated NEP $=1.67 \times 10^{-11} \mathrm{~W} / \sqrt{ } \mathrm{Hz}$ of our earlier detector working at $10 \mathrm{~K}$ reported in [10] was for the imaging system noise rather than for the HTS Josephson detector as it was amplified 100x and included the lock-in amplifier noise. Using the pre-amplified noise figure, i.e., $1 \mu \mathrm{V} / 100=10 \mathrm{nV}$, a NEP of $\sim 1.67 \times 10^{-13} \mathrm{~W} / \sqrt{ } \mathrm{Hz}$ was btained for the detector reported in [10]. 


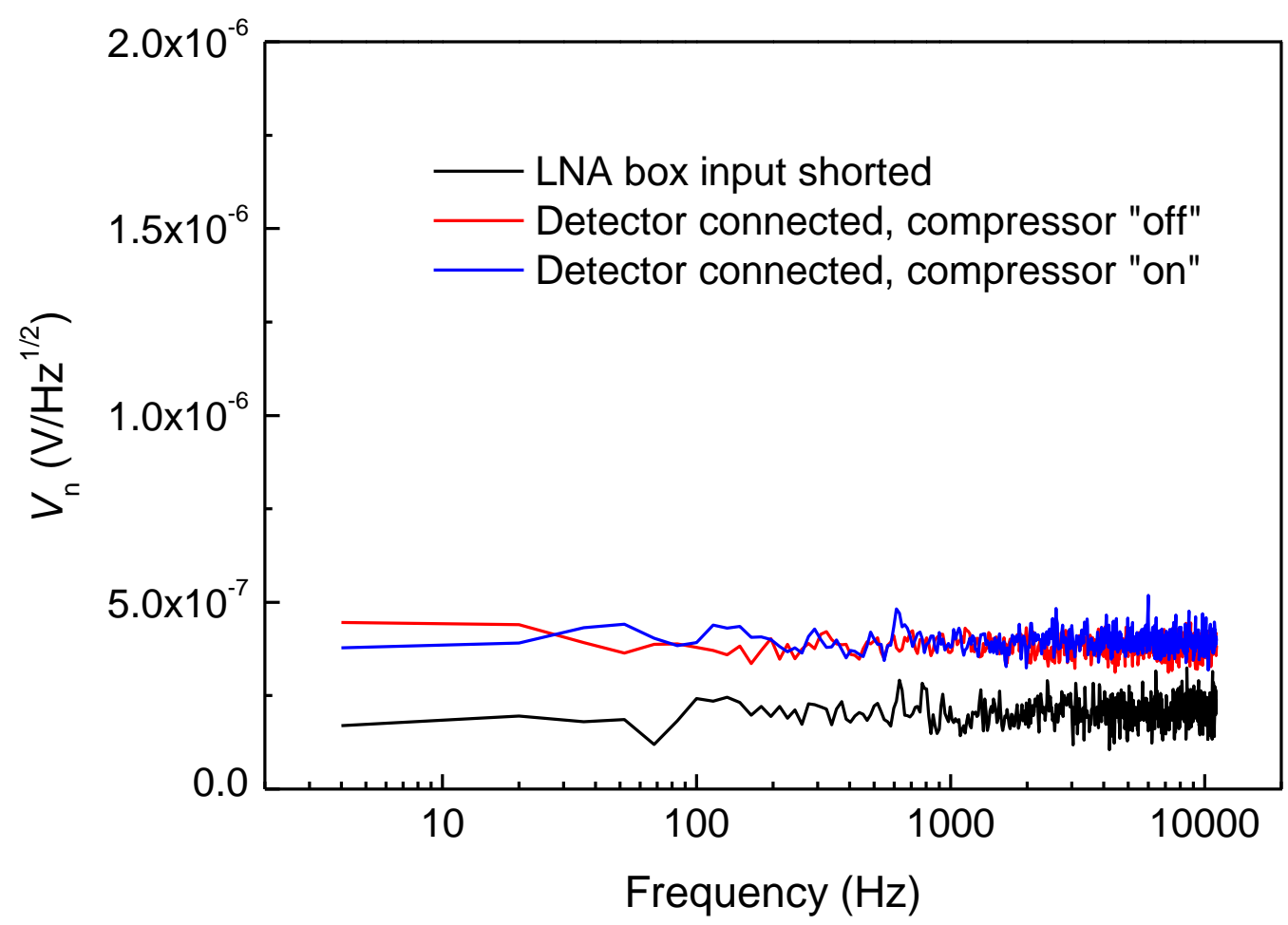

Figure 8: Noise spectra of the LNA only and connected to the detector in the cryocooler with compressor "off" and "on" when the THz signal is blocked.

\section{THz Imaging Results}

Figure 9 shows a THz image (left) at $614.2 \mathrm{GHz}$ of a pair of scissors in a purse (right) obtained with the cryocooled HTS Josephson detector at $70 \mathrm{~K}$. It demonstrates the ability of THz radiation to see through non-conducting packaging material and reveal the concealed metal item. The image scanning time was 10 mins for the image size of $100 \times 100$ pixels with one detector (single pixel). Scaling up to arrays of such detectors would reduce the scanning time to suit application like realtime security screening. Initial design and test of a proof-of-concept HTS Josephson junction $\mathrm{THz}$ detector array was reported [18]. 


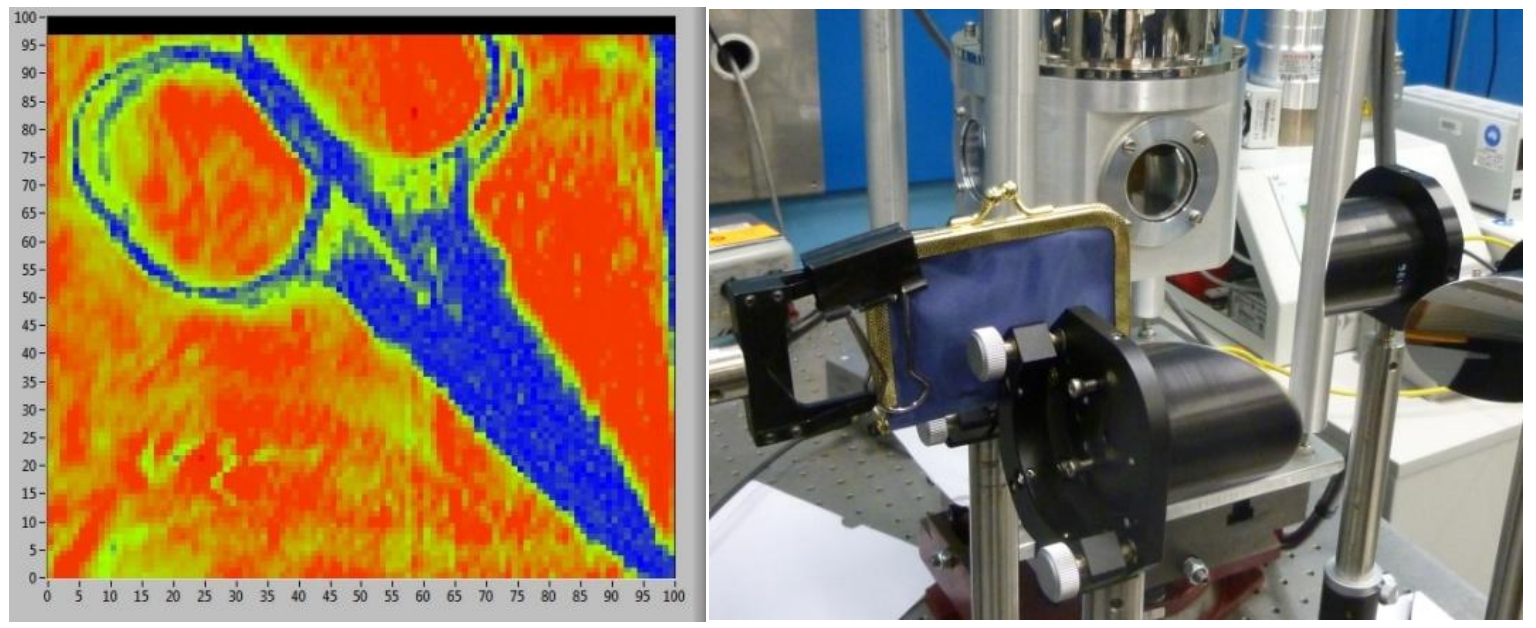

Figure 9: A THz image (left) of a pair of scissors in a purse (right) obtained with the cryocooled HTS Josephson detector at $70 \mathrm{~K}$.

Figure 10 shows a THz transmission image of a leaf obtained by the cryocooled HTS detector operating at $77 \mathrm{~K}$ and $60 \mathrm{~K}$. The imaging resolutions and times (10 mins) for Figs. 10 (b) and (c) were identical. The image highlights one of the key $\mathrm{THz}$ features, i.e., the sensitivity to water with the higher water content in the leaf veins exhibiting higher attenuation. The $77 \mathrm{~K}$ image is noisier than that of the $60 \mathrm{~K}$ image. The $60 \mathrm{~K}$ image shows very clear details of the small variations in the leaf's thickness and minor vein details as a result of a higher SNR.

(a)

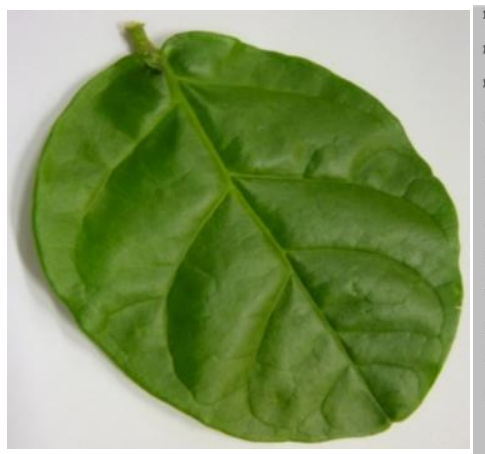

(b)

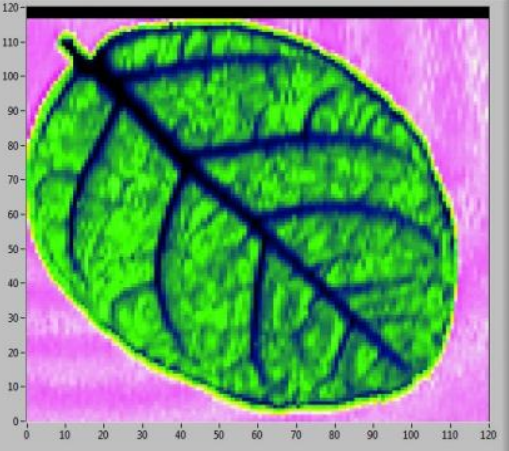

(c)

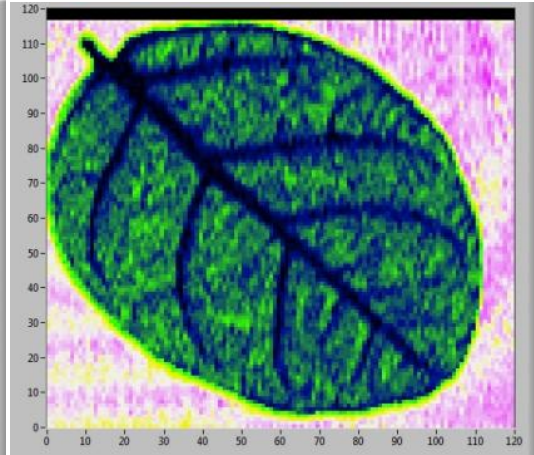

Figure 10: The photograph (a) and THz images of a leaf obtained with the Josephson detector operating at $60 \mathrm{~K}$ (b) and $77 \mathrm{~K}(\mathrm{c})$.

\section{Conclusions:}

A cryogen-free HTS Josephson detector-based terahertz imager was presented. A compact, simplified solid-state device based quasi-optic THz imaging system at $600 \mathrm{GHz}$ band was demonstrated and high-quality $\mathrm{THz}$ images were obtained. The DC and AC characteristics of the thinfilm antenna-coupled HTS step-edge junction detector were studied against the operating 
temperatures from $50 \mathrm{~K}$ to $77 \mathrm{~K}$, a temperature range attainable with a relatively cheap more compact single stage cryocooler. The detector responsivity, SNR and NEP improved dramatically with decreasing temperature; a sensitivity of $\sim 7000 \mathrm{~V} / \mathrm{W}, \mathrm{SNR} \sim 1 \times 10^{5}$ and a NEP $\approx 3 \times 10^{-13} \mathrm{~W} / \mathrm{V} \mathrm{Hz}$ were obtained at $50 \mathrm{~K}$. The quality of the acquired images improves significantly with decreasing temperature due to improved responsivity and SNR, a clear advantage of using a cryocooler rather than liquid nitrogen for cooling. The results clearly demonstrated the potential of terahertz imaging using a cryogen-free HTS Josephson junction detector, an important step for gaining industry acceptance of this technology.

\section{ACKNOWLEDGMENT}

Earlier contributions from Drs Andrew Hellicar, Nasiha Nikolic, John Macfarlane, and the HTS chip fabrication by Jeina Lazar of CSIRO are gratefully acknowledged.

\section{References:}

1. Tonouchi M 2007 Cutting-edge terahertz technology, Nature Photonics 1, 97-105.

2. Jansen C, et al. 2010 Terahertz imaging: applications and perspectives, Applied Optics. 49 (19), E48.

3. Chan W L, Deibel J, and Mittleman D M 2007 Imaging with terahertz radiation, Rep. Prog. Phys. 70 1325-1379.

4. Mathanker S K, Weckler P R and Wang N 2013 Terahertz (THz) applications in food and agriculture: a review, Transactions of the ASABE. 56 (3) 1213-1226.

5. Ozyuzer, L, et al. 2007 Emission of coherent THz radiation from superconductors, Science 318, 1291-1293.

6. Wang H B, et al. 2010 Coherent terahertz emission of intrinsic Josephson junction stacks in the hot spot regime, Phys. Rev. Lett. 105057002.

7. Ariyoshi S, Otani C, Dobroiu A, Sato H, Kawase K, Shimizu H M, Taino T, and Matsuo H 2006 Terahertz imaging with a direct detector based on superconducting tunnel junctions Appl. Phys. Lett. 88203503.

8. Luukanena A, Gronberg L, Helisto P, Penttila J S, Seppa H, Sipola H, Dietlein C R and Grossman E N 2006 An array of antenna-coupled superconducting microbolometers for passive indoors realtime THz imaging Proc. of SPIE 621262120 Y-2.

9. Chen J, Kurigata Y, Wang H B, Nakajima K, Yamashita T and Wu P H 2003 Wideband frequency metrology using high temperature superconducting Josephson junctions IEEE Trans. Appl. Supercond. 13 1143-1146. 
10. Du J, Hellicar A D, Li L, Hanham S M, Nikolic N, Macfarlane J C and Leslie K E 2008 Terahertz imaging using a high-Tc superconducting Josephson junction detector Supercond. Sci. Technol. 21 125025.

11. Du J, Hellicar A D, Li L, Hanham S, Macfarlane J C, Leslie K E, Nikolic N, Foley C P, and Greene K J 2009 Terahertz imaging at 77 K Supercond. Sci. Technol. 22114001.

12. Du J, Hellicar A D, Hanham S, Li L, Macfarlane J C, Leslie K E and Foley C P 2011 Terahertz and millimetre wave imaging with a broadband Josephson detector working above $77 \mathrm{~K} J$ Infrared, Millimeter, and Terahertz Waves 32 681-690.

13. Foley C P, Mitchell E E, Lam S K H, Sankrithyan B, Wilson Y M, Tilbrook D L, and Morris S J 1999 Fabrication and characterisation of YBCO single grain boundary step edge junctions IEEE Trans. Appl. Supercond. 9 4281-4284.

14. Mitchell E E and Foley C P 2010 YBCO Step-edge junctions with high IcRn Supercond. Sci. Technol 23065007.

15. Du J, Lam S K H, and Tilbrook D L 2001 Metallization and interconnection of HTS YBCO thin film devices and circuits, Supercon. Sci. \& Technol 14 820-825.

16. Van Duzer T and Turner C W 1999 Superconductive Devices and Circuits, 2nd edition, PrenticeHall, Inc, New Jersey, USA.

17. Hesler J L and Crowe T W 2007 NEP and responsivity of THz zero-bias Schottky diode detectors, the $15^{\text {th }}$ Intern. Conf. on Terahertz Electronics IRMMW-THz, September 2007 pp 844-5. Also see

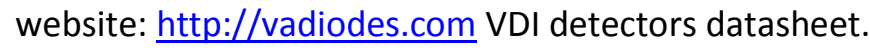

18. Du J, Hellicar A D, Leslie K E, Nikolic N, Hanham S, Macfarlane J C, Foley C P 2013 Towards Large Scale HTS Josephson Detector Arrays for THz Imaging, Supercond. Sci \& Technol. 26 115012. 